\title{
Canadian Hydrogen Intensity Mapping Experiment (CHIME) Pathfinder
}

Kevin Bandura $^{a}$, Graeme E. Addison ${ }^{b}$, Mandana Amiri ${ }^{b}$, J. Richard Bond ${ }^{c d}$, Duncan Campbell-Wilson ${ }^{e}$, Liam Connor ${ }^{c f}$, Jean-François Cliche ${ }^{a}$, Greg Davis ${ }^{b}$, Meiling Deng ${ }^{b}$, Nolan Denman $^{f}$, Matt Dobbs ${ }^{a}$, Mateus Fandino ${ }^{b}$, Kenneth Gibbs $^{b}$, Adam Gilbert ${ }^{a}$, Mark Halpern ${ }^{b}$, David Hanna $^{a}$, Adam D. Hincks ${ }^{b}$, Gary Hinshaw ${ }^{b}$, Carolin Höfer ${ }^{b}$, Peter Klages ${ }^{f g}$, Tom L. Landecker $^{h}$, Kiyoshi Masui ${ }^{b}$, Juan Mena ${ }^{a}$, Laura B. Newburgh ${ }^{i}$, Ue-Li Pen ${ }^{c}$, Jeffrey B. Peterson $^{j}$, Andre Recnik ${ }^{f}$, J. Richard Shaw ${ }^{c}$, Kris Sigurdson ${ }^{b}$, Michael Sitwell ${ }^{b}$, Graeme Smecher $^{a}$, Rick Smegal ${ }^{b}$, Keith Vanderlinde ${ }^{f i}$, and Don Wiebe ${ }^{b}$

${ }^{a}$ Department of Physics, McGill University, 3600 University St, Montreal, Canada

${ }^{b}$ Department of Physics and Astronomy, University of British Columbia, 6224 Agricultural Rd. Vancouver, V6T 1Z1, Canada

${ }^{c}$ CITA, 60 St George St, Toronto, ON, M5S 3H8, Canada

${ }^{d}$ Canadian Institute for Advanced Research, CIFAR Program in Cosmology and Gravity, Toronto, ON M5G 1 Z8

eSydney Institute for Astronomy, School of Physics, University of Sydney, NSW 2006, Australia

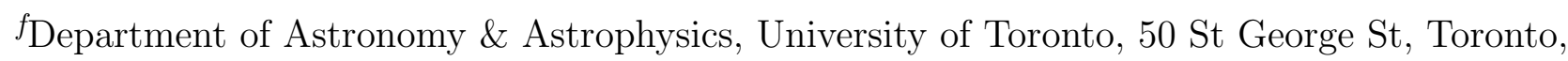
ON, M5S 3H4, Canada

${ }^{g}$ IBM Canada

${ }^{h}$ National Research Council Canada, Dominion Radio Astrophysical Observatory, Box 248, Penticton BC V2A 6J9 Canada

${ }^{i}$ Dunlap Institute for Astronomy \& Astrophysics, University of Toronto, 50 St George St, Toronto, ON, M5S 3H4, Canada

${ }^{j}$ McWilliams Center for Cosmology, Carnegie Mellon University, Department of Physics, 5000 Forbes Ave, Pittsburgh PA 15213, USA

\begin{abstract}
A pathfinder version of CHIME (the Canadian Hydrogen Intensity Mapping Experiment) is currently being commissioned at the Dominion Radio Astrophysical Observatory (DRAO) in Penticton, BC. The instrument is a hybrid cylindrical interferometer designed to measure the large scale neutral hydrogen power spectrum across the redshift range 0.8 to 2.5. The power spectrum will be used to measure the baryon acoustic oscillation (BAO) scale across this poorly probed redshift range where dark energy becomes a significant contributor to the evolution of the Universe. The instrument revives the cylinder design in radio astronomy with a wide field survey as a primary goal. Modern low-noise amplifiers and digital processing remove the necessity for the analog beamforming that characterized previous designs. The Pathfinder consists of two cylinders $37 \mathrm{~m}$ long by $20 \mathrm{~m}$ wide oriented northsouth for a total collecting area of 1,500 square meters. The cylinders are stationary with no moving parts, and form a transit instrument with an instantaneous field of view of $\sim 100$ degrees by 1-2 degrees. Each CHIME
\end{abstract}

Send correspondence to K.Bandura: E-mail: kevin.bandura@mcgill.ca 
Pathfinder cylinder has a feedline with 64 dual polarization feeds placed every $\sim 30 \mathrm{~cm}$ which Nyquist sample the north-south sky over much of the frequency band. The signals from each dual-polarization feed are independently amplified, filtered to $400-800 \mathrm{MHz}$, and directly sampled at $800 \mathrm{MSps}$ using 8 bits. The correlator is an FX design, where the Fourier transform channelization is performed in FPGAs, which are interfaced to a set of GPUs that compute the correlation matrix. The CHIME Pathfinder is a $1 / 10$ th scale prototype version of CHIME and is designed to detect the BAO feature and constrain the distance-redshift relation.

The lessons learned from its implementation will be used to inform and improve the final CHIME design.

Keywords: CHIME, cosmology, SPIE Proceedings, Intensity Mapping, BAO

\section{INTRODUCTION}

The nature of the dark energy that drives the accelerated expansion of the Universe is one of the greatest mysteries in modern science. Of the observational techniques that probe dark energy 1 one of the most promising is measuring the baryon acoustic oscillation (BAO) scale using fluctuations in the large-scale distribution of neutral hydrogen.2]

The BAO feature was imprinted in the matter correlation function at a scale of approximately 150 comoving $\mathrm{Mpc}$ when baryons decoupled from radiation. By measuring the BAO standard ruler in the large-scale structure across redshift, the expansion history of the universe is measured. In particular the period from redshift 1-2 has the most power to distinguish between dark energy models. The first clear detection of BAO came from analyzing the Sloan Digital Sky Survey (SDSS) luminous red galaxies at redshift z $\sim 0.35{ }^{3}$ The detection was verified in the two degree field galaxy redshift survey at redshift $\sim 0.2$. ${ }^{4}$ It has more recently been measured at three redshifts of $\sim 0.44, \sim 0.6$, and $\sim 0.7$ in the WiggleZ Dark Energy Survey and Baryon Oscillation Spectroscopic Survey $(\mathrm{BOSS})^{\sqrt{6}}$ at $z \sim 0.57$. BAO were further detected in the Ly- $\alpha$ forest at redshift $\sim 2.4$ with BOSS $7 \sqrt{8}$

The $21 \mathrm{~cm}$ neutral hydrogen emission is an accurate tracer of matter on cosmological scales ${ }^{9}[1]$ The isolation of the $21 \mathrm{~cm}$ line eliminates the spectral confusion handicapping optical surveys operating at redshifts of 1 to 3 . Since the BAO angular scale is on the order of a degree on the sky, the detection of individual galaxies as in an optical BAO survey is not necessary. Instead, a $21 \mathrm{~cm}$ intensity map measures the aggregate $21 \mathrm{~cm}$ emission at larger scales. These features make neutral hydrogen an excellent tracer of large scale structure and BAO at the redshifts where dark energy becomes dominant.

The properties of the instrument are set to measure the BAO scale. The BAO first peak corresponds to an angular size of $1.35^{\circ}$ at $z=2.5$ and $3^{\circ}$ at $z=0.8$. Nyquist sampling the BAO feature in a $21 \mathrm{~cm}$ map requires baseline lengths of $15 \mathrm{~m}$ and $63 \mathrm{~m}$ at the corresponding redshifts. Along the line of sight, at redshift 0.8 $(800 \mathrm{MHz})$ the BAO scale corresponds to a correlation at $20 \mathrm{MHz}$ separation, and at redshift $2.5(400 \mathrm{MHz})$ it corresponds to a $12 \mathrm{MHz}$ separation correlation.

In order to measure the BAO in neutral hydrogen, we are building a close-packed interferometer radio telescope, the Canadian Hydrogen Intensity Mapping Experiment (CHIME) Pathfinder. It is a smaller scale version of the full CHIME instrument and is being built to inform the full CHIME design in addition to making competitive measurements of the BAO scale. The pathfinder is comprised of two cylinders $20 \mathrm{~m}$ wide by $37 \mathrm{~m}$ long which are set $2 \mathrm{~m}$ apart. The cylinders are oriented along the north-south direction and fixed. The cylindrical nature of the structure gives the instrument a $\sim 100^{\circ}$ instantaneous field of view in the north-south direction, allowing a full survey of half the sky each sidereal day as the earth rotates. The close-packed configuration gives the instrument a high intensity mapping speed.

A summary of the Pathfinder specifications is in Table 1. All components have been designed with an eye toward scaling the instrument by a factor of ten. The CHIME Pathfinder has 128 dual-polarization feeds and processes $400 \mathrm{MHz}$ of bandwidth. Scaling to the 1280 feeds required for the CHIME telescope is made affordable today by advances in analog and digital processing. We have designed low-cost low-noise amplifiers based on commercial components that achieve a noise figure of $35 \mathrm{~K}$ at room temperature. This drastically simplifies the cylinder feed line receiver design and number of components. For the channelizer we use field-programmable gate array (FPGA) processors which offer many digital signal processing blocks as well as many high-speed serial links, greatly simplifying the design of the digital network required to re-arrange and redistribute all the data 


\begin{tabular}{l|c}
\hline Frequency Range & $400 \mathrm{MHz}-800 \mathrm{MHz}$ \\
\hline Redshift Range & $2.5-0.8$ \\
\hline Beam Size & $1^{\circ}-0.5^{\circ}$ \\
\hline Frequency Resolution & $390 \mathrm{kHz}, 1024 \mathrm{bins}$ \\
\hline E-W FoV & $2.5^{\circ}-1.3^{\circ}$ \\
\hline N-S FoV & $\sim 100^{\circ}$ \\
\hline Single Source Observing Time per Day & Equator: $10 \mathrm{~min}-5 \mathrm{~min}$ \\
& $45^{\circ}: 14 \mathrm{~min}-7 \mathrm{~min}$ \\
& NCP: $24 \mathrm{hr}$ \\
\hline Structure & 2 cylinders $-37 \mathrm{~m} \mathrm{x} 20 \mathrm{~m}$ \\
\hline Number of Beams & 128 dual polarization \\
\hline Receiver Noise Temperature & $50 \mathrm{~K}$ \\
\hline
\end{tabular}

Table 1. Pathfinder design parameters

for correlation. Our use of graphics processing units (GPU) for the correlation allows for a rapid development cycle and unique additional processing.

The layout of the paper is as follows. Section 2 describes the science goals the CHIME Pathfinder hopes to attain. Section 3 describes the mechanical structure of the telescope, Section 4 describes the details of the feed and analog electronics and Section 5 describes the digital backend of the CHIME Pathfinder.

\section{SCIENCE GOALS}

The CHIME Pathfinder will measure the large-scale structure of the Universe from redshift $0.8-2.5$ in $21 \mathrm{~cm}$ emission. Though the matter power spectrum contains a wealth of useful information, we are primarily interested in measuring the BAO, relics of primordial sound waves that produce enhanced correlation at a characteristic scale. The physical observables corresponding to the BAO scale in the radial (redshift) and transverse (angular) directions are $\Delta z=H(z) r_{s} / c$ and $\Delta \theta=r_{s} / D_{M}(z)$, where $H(z)$ is the Hubble parameter, $r_{s}$ is the sound horizon at baryon-photon decoupling, and $D_{M}(z)$ is the transverse comoving distance. The sound horizon is wellconstrained by Cosmic Microwave Background temperature anisotropy measurements, ${ }^{12} 13$ allowing measurement of the BAO scale as a function of redshift to directly probe the distance-redshift relation and expansion history of the Universe.

A major challenge faced by hydrogen intensity mapping experiments is the presence of bright astrophysical radio emission at the same frequencies as the cosmological $21 \mathrm{~cm}$ emission we are searching for. These foregrounds are dominated by synchrotron emission from both our own galaxy and high redshift radio galaxies. They have brightness temperatures up to $700 \mathrm{~K}$, many orders of magnitude brighter than the $0.1 \mathrm{mK}$ signal we are looking for.

Fortunately the synchrotron emission is spectrally very smooth, and can be separated on this basis from the large-scale structure $21 \mathrm{~cm}$ signal. Though this is superficially straightforward, instrumental realities such as frequency-dependent beams and polarization leakage present added challenges, which can be surmounted with precise calibration 14 and new analysis techniques. ${ }^{15}$ In Figure 1 we demonstrate the analysis of simulated time stream data, showing how we can effectively remove foregrounds using a Karhunen-Loève transform to separate the signals based on their statistics.

The primary quantity we are interested in measuring is the spatial power spectrum of the large-scale structure, which contains most of the useful cosmological information. Unfortunately the removal of foreground contamination inevitably reduces our sensitivity to the signal we are interested in. Using the $m$-mode formalism, the two-dimensional matter power spectrum can be reconstructed in the presence of foregrounds 15 16 As expected we lose sensitivity to the largest scale fluctuations in the line-of-sight direction, but still retain significant sensitivity to the peaks of the BAO. 


\section{Unpolarised Foreground $\quad$ Polarised Foreground (Q) 21cm Signal}
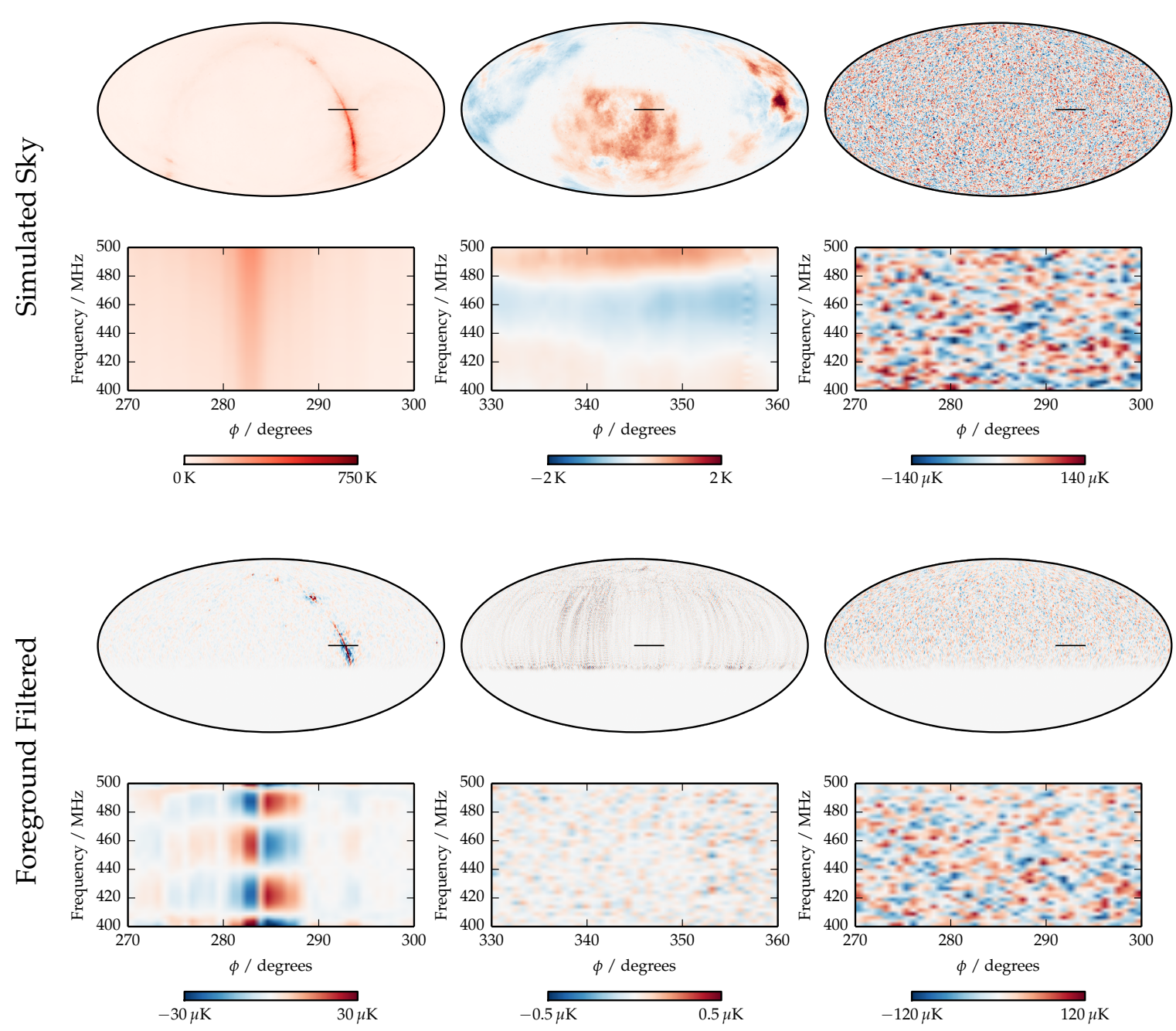

Figure 1. This plot illustrates the process of foreground removal on simulations of the radio sky. The top row of plots shows sky maps of the individual components: unpolarized foregrounds, polarized foregrounds (showing Stokes Q only), and the $21 \mathrm{~cm}$ signal. On the bottom row we show the maps we would make after foreground cleaning visibilities from the CHIME Pathfinder. In each, the upper panel shows a frequency slice at $400 \mathrm{MHz}$, and the lower panel a slice through the galactic plane from 400 to $500 \mathrm{MHz}$. Both the polarized and unpolarized foregrounds become substantially suppressed, whereas the $21 \mathrm{~cm}$ signal is largely unaffected. This leaves a clear correspondence between the original signal simulation and the foreground subtracted signal, while leaving the foreground residuals over 10 times smaller in amplitude than the signal. Figure from Shaw et. al. 2014!

In Figure 2 we illustrate the forecast constraints that the CHIME Pathfinder could place on the expansion history though measuring the BAO. The figure illustrates the forecast Pathfinder statistical limit of $D_{V}(z)=$ $\left[D_{M}(z)^{2} \frac{c z}{H(z)}\right]^{1 / 3}$ with two years observation time in comparison to current measurements.

\subsection{Ancillary Science}

The CHIME Pathfinder is an excellent platform to pursue ancillary science goals due to its design and operating parameters. Some of those potential goals are listed below: 


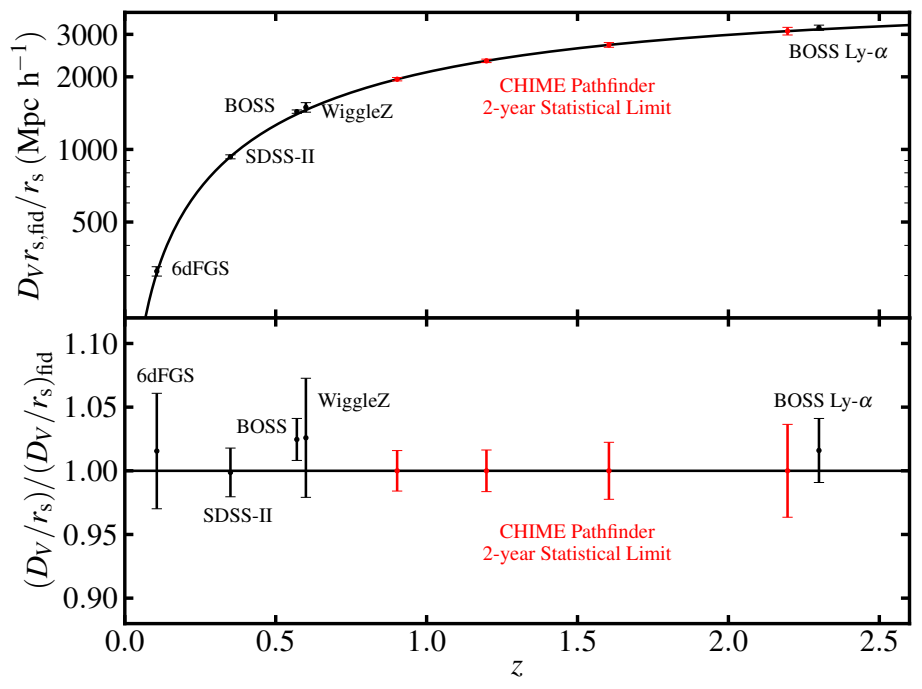

Figure 2. Forecast CHIME Pathfinder constraints on the expansion history parameterized using the ratio of $D_{V}$ (see text) to the sound horizon $r_{s}$ as a function of redshift, shown relative to a fiducial $\Lambda$ CDM cosmology with $h=0.7, \Omega_{\Lambda}=0.7$,

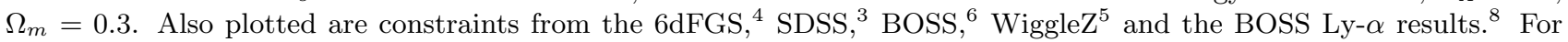
the BOSS Ly- $\alpha$ results the error bars are just a direct fractional error translation from the $\alpha_{i s o}$ constraints for illustrative purposes, and should not be interpreted as their constraint on $D_{V}$. For the CHIME Pathfinder, the forecast error bars were calculated using the methods from Shaw et. al. $2014^{15}$ and represent the statistical limit with two years integration time.

Pulsars: The CHIME Pathfinder's wide field of view lends itself to the monitoring of pulsar timing. The telescope is able to see every pulsar in the northern hemisphere for at least $\sim 5$ minutes each day. Every day hundreds of pulsars can be observed and their dispersion measure, timing and scintillation can be monitored. An additional backend is being built which will receive full rate data on individual sky beams for measurement of pulse arrival times for a list of known pulsars.

Radio transients: The survey nature of the CHIME Pathfinder also lends itself to serendipitous detection of radio transients, including fast radio bursts $17[19$ The CHIME Pathfinder has the sensitivity and field of view to discover many undetected transient sources. An exciting possible transient source is the radio afterglow from compact binary coalescence. $\frac{20}{20}$

Galactic polarization and Rotation Measure: In order to make a precise intensity map of the sky, the CHIME Pathfinder must be able to remove the foreground polarized emission from the galaxy. The CHIME Pathfinder will produce an accurate map of foreground polarization, which can be used to further understand Galactic magnetic fields.

Cosmic rays: The close-packed nature of the CHIME Pathfinder will allow for the telescope to detect the radio emission from cosmic-ray air showers. Information about the air shower can be reconstructed from the arrival time, intensity, and polarization of the air-shower pulse.

\section{TELESCOPE}

The CHIME telescope structure consists of two adjacent parabolic cylinders, with a ground plane, feeds, and low noise amplifiers held along the focal line of each cylinder beneath a walkway, see Figure 3a. The reflective surface is galvanized steel mesh bolted to the underlying structure. The cylinder axes run north-south.

Each cylinder is $20 \mathrm{~m}$ across and $37 \mathrm{~m}$ long, see Figure 3c. The focal length of the parabola is $5 \mathrm{~m}$ and the telescopes have a focal ratio of $\mathrm{f} / 0.25$. The telescope was designed to rely on standard steel construction 
practices as much as possible. On-site welding is held to an absolute minimum to avoid RF disturbance of the host observatory. The structure consists of parabolic steel trusses set every $5 \mathrm{~m}$ north-south supported by cement feet which bear below the frost line. Purlins spaced $1 \mathrm{~m}$ apart run parallel to the cylinder axis, bolted to each truss. The structure is not very different from that of any warehouse roof, except that the east-west cross section is parabolic, the roof itself is instead mesh, and there is no floor. The structure is designed to have a peak deformation of less than $2 \mathrm{~cm}$ under a wind and snow load of $1 \mathrm{kN} / \mathrm{m}^{2}$.

The mesh properties have been chosen as a compromise between reflectivity to radio wave and the ability to shed snow. CHIME has no moving parts and so, unlike a conventional radio telescope, it can not be tipped over to shed accumulated snowfall. A course mesh allows snow to fall through, which avoids loss of observation time due to perturbed reflectivity. However, a course mesh also transmits more thermal radiation from the ground, raising the system temperature. The noise level from leakage of $300 \mathrm{~K}$ radiation through mesh is plotted in Figure $3 \mathrm{~d}$ across the CHIME band for two different mesh options. The CHIME Pathfinder uses $19 \mathrm{~mm}(3 / 4$ in) spacing 14 Birmingham Wire Gauge (BWG) mesh, which is compared to a $25 \mathrm{~mm}$ (1 in) 10 BWG mesh. These are the heaviest wire sizes available at each spacing and thus the most reflective mesh at each size. The noise contribution of the $19 \mathrm{~mm}$ mesh is roughly $1 \mathrm{~K}$ lower across the upper half of the CHIME passband, which corresponds to a sensitivity difference per year equivalent to 15 days of extra observations. Over the 2013-14 winter snow was on the CHIME mesh only immediately after the largest snow falls, for a total of four days. The mesh is available in rolls of $2 \mathrm{~m}$ width. The $1 \mathrm{~m}$ purlin spacing is chosen so that the mesh sits on three purlins and takes the shape of the reflector. The mesh is riveted every $75 \mathrm{~cm}$ along the purlin.

Photogrammetry has been used to measure the shape of the steel support structure. The results are shown in Figure $3 \mathrm{~b}$ as deformations with respect to the nominal design shape. The rms deformation here is $5.2 \mathrm{~mm}$. Deformation of the mesh with respect to the steel structure has been measured by hand at hundreds of points across the structure. The mesh surface error is $1.4 \mathrm{~cm} \mathrm{rms}$ and dominates the telescope surface error budget. This rms gives a cylinder efficiency of $99 \%$ and $97 \%$ at $400 \mathrm{MHz}$ and $800 \mathrm{MHz}$ respectively.

\section{ANALOG CHAIN}

The analog system of the CHIME Pathfinder consists of the components shown in Figure 4. The feeds and LNAs are located along the focal line. The signals are then sent over coaxial cables to a shielded RF enclosure. The signals are then bandpass filtered and further amplified to achieve an input power of $-21 \mathrm{dbm}$ at the input of the ADCs. The overall receiver temperature design for CHIME is $50 \mathrm{~K}$. This includes ground effects but does not contain any contribution from the sky. The analog components are described in more detail in the sections below.

\subsection{Feed}

The CHIME feed is a clover-leaf shaped compact dual-polarization feed. It is a modification of the four-square feeds developed for the Molonglo Observatory ${ }^{21}$ The feed petals, balun stem, and support base are all made from printed circuits boards (PCB), as shown in Figure 5a. As shown in Figure 5b, the petals have curved outer edges that broaden the frequency response by reducing the number of individual resonant dimensions. The

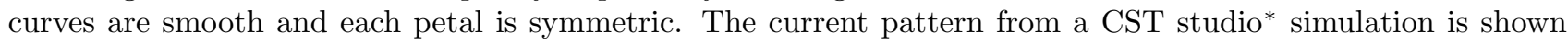
in Figure $5 \mathrm{c}$ for one linear polarization at $600 \mathrm{MHz}$. The currents near the gaps between petals run in opposing directions so they cancel, and do not contribute to the radiation pattern. For this polarization, far-field radiation arises from the coherent currents running along the curved outer edges of the top and bottom pair of petals.22

Differential signals from pairs of adjacent petals are combined through tuned baluns to form one singleended output. Thus each single polarization signal involves currents in all four petals. Full baluns, from both polarizations, consist of four identical microstrip transmission lines along four vertical support boards (the "stem") and a horizontal base board. Both of the single-ended outputs are on the base board. Each transmission line is varied in several abrupt steps, and the lengths and characteristic impedances of the transmission line segments are carefully tuned to match impedance. Electrical losses in conventional circuit board materials

\footnotetext{
*https://www.cst.com/
} 


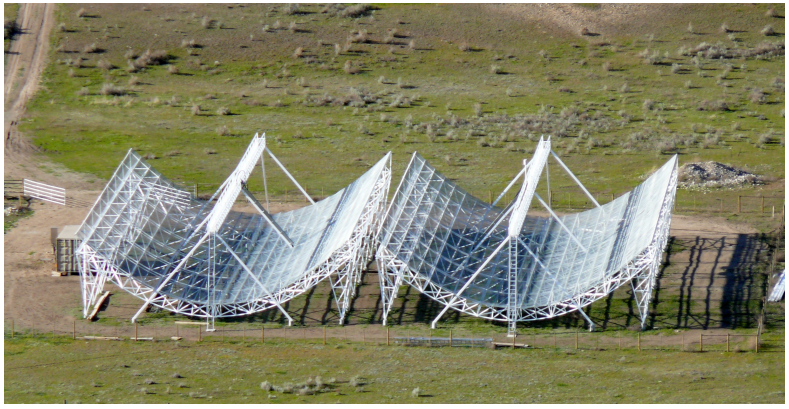

(a)

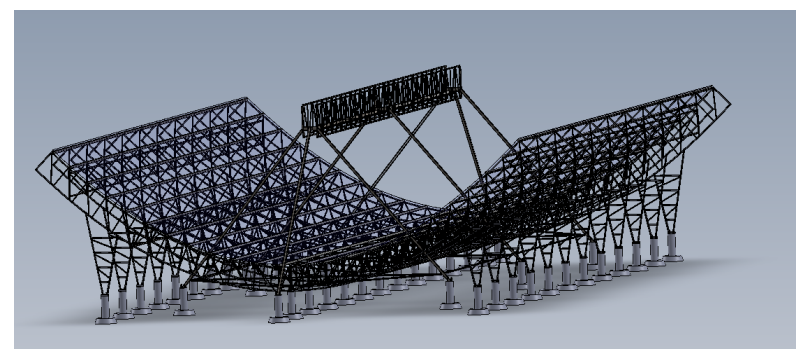

(c)

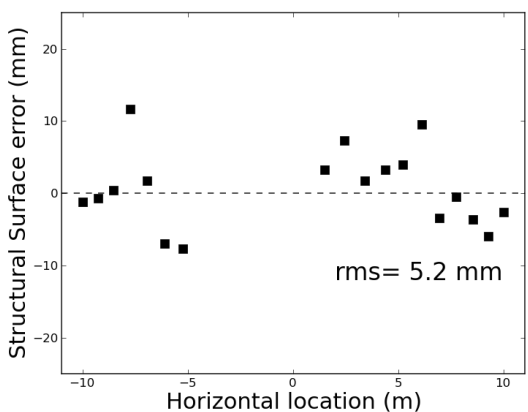

(b)

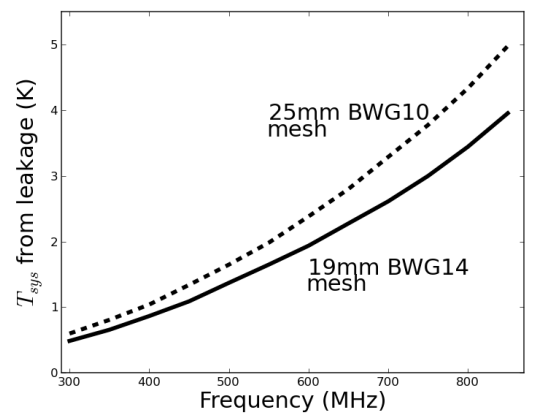

(d)

Figure 3. (a) Photograph of the CHIME Pathfinder at the Dominion Radio Astrophysical Observatory (DRAO), April 2014. The digital correlator is housed in an RFI enclosure built into the sea container visible at the left hand (western) edge of the western cylinder. Signals from the feeds are brought down the middle diagonal support legs and across the the correlator under the reflectors. Removable ladders visible at the south end of each focal line allow access. (b) Steel support structure normal surface error of the western cylinder obtained from photogrammetry is plotted in millimeters. The quantity plotted is deviation of the supports from the design shape, not from a best fit parabola. The mesh has been measured to have a shape deviation of $14 \mathrm{~mm} \mathrm{rms} \mathrm{with} \mathrm{respect} \mathrm{to} \mathrm{the} \mathrm{steel} \mathrm{support} \mathrm{structures} \mathrm{for} \mathrm{the} \mathrm{surface} \mathrm{mesh.} \mathrm{(c)} \mathrm{A}$ CAD model of the telescope support structure. The cement base of each support sits $70 \mathrm{~cm}$ below grade to avoid possible frost heave. Each truss is supported on three legs. Each focal line sits on seven legs, with the northern leg only serving to lock out a north-south parallelogram motion of the six other legs. The support legs are $10 \mathrm{~cm} \times 15 \mathrm{~cm}$ hollow steel structures and pass above the instrument ground plane, allowing the one-dimensional feed array to run uninterrupted along the focal line. (d) Frequency-dependent noise leakage of an assumed $300 \mathrm{~K}$ load at normal incidence. The calculations have been performed for mesh with $19 \mathrm{~mm}$ spacing made of BWG 14 wire and $25 \mathrm{~mm}$ spacing, BWG 10 wire. These are the heaviest wire gauges we could find in mesh of these spacings. The comparatively dry snow falling at $590 \mathrm{~m}$ elevation in the BC interior typically falls through the $19 \mathrm{~mm}$ mesh from which CHIME is built. 


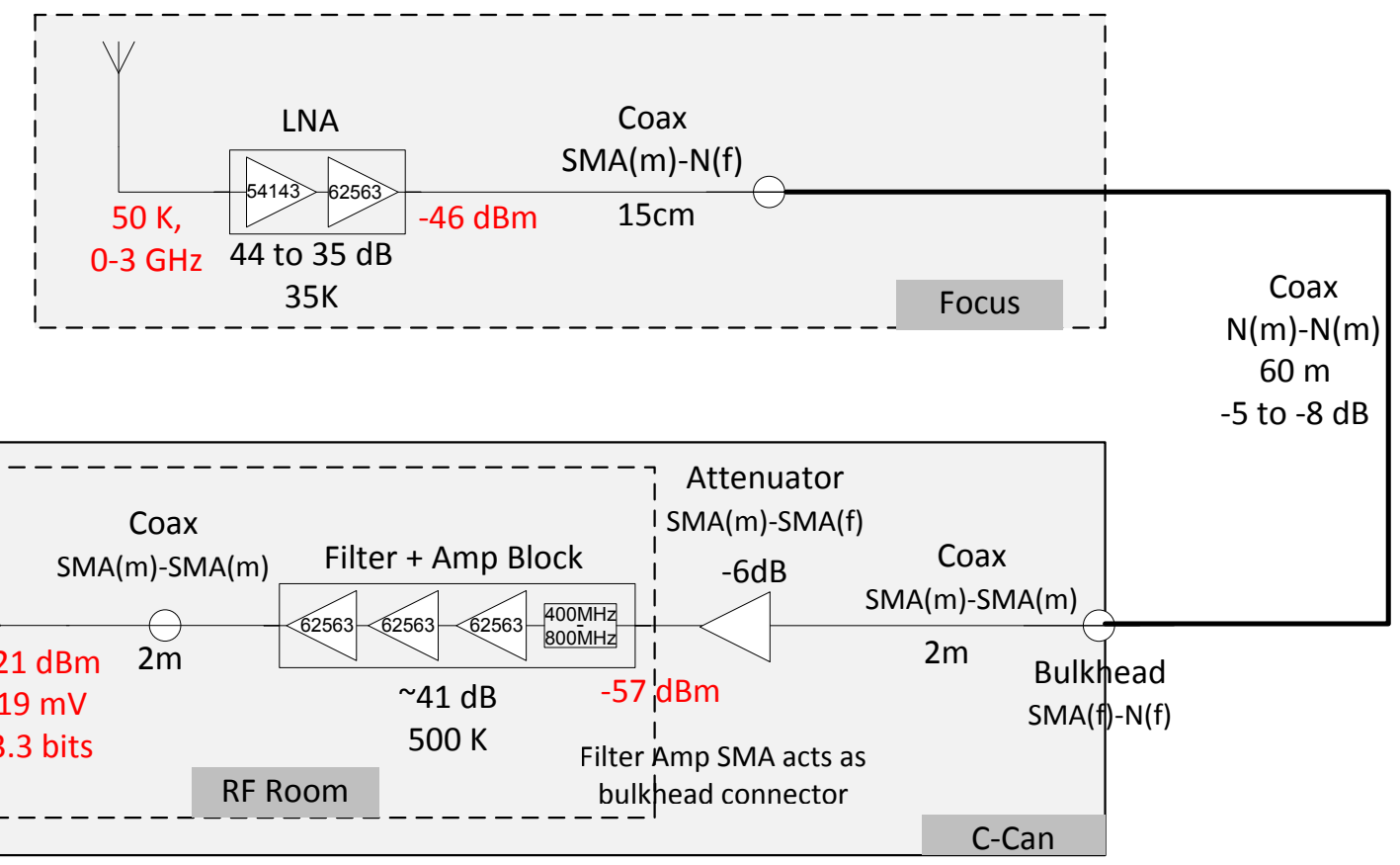

Figure 4. The analog system signal chain overview. The clover-leaf feed receives the sky signal. The received signal is then amplified by the low-noise amplifier block by 35 to $44 \mathrm{~dB}$ across the $400-800 \mathrm{MHz}$ band while adding $\sim 35 \mathrm{~K}$ noise. The signal is then transmitted over $60 \mathrm{~m}$ of LMR-400 coaxial cable to a central RF-shielded enclosure. The signal is attenuated there by approximately $6 \mathrm{~dB}$, customized to each amplifier chain. The signal is then bandpass filtered to $400-800 \mathrm{MHz}$ and further amplified by $\sim 41 \mathrm{~dB}$ to achieve an input to the ADC of $-21 \mathrm{dBm}$ power.

generate unacceptable loss for astronomical instrumentation, so Teflon-based PCB is used for both the balun stem and support base. Between petals the PCB is completely removed to minimize loss along the slot transmission.

The measured returned loss compared with simulation is shown in Figure 6a, and the measured beam pattern is shown in Figures $6 \mathrm{~b}$ and $6 \mathrm{c}$

\subsection{LNA}

The low-noise amplifier (LNA) is located at the focus and is directly attached to the feed. The LNA has two gain stages. The first is based on an Avago $54143 \mathrm{GaAs}$ enhancement mode pseudomorphic high electron mobility transistor (E-pHEMT). It is followed by an Avago MGA-62563 E-pHEMT radio frequency integrated circuit (RFIC). The achieved noise figure with this design is $35 \mathrm{~K}$ across most of the band, as shown in Figure $7 \mathrm{~d}$.

The input matching, output matching and feedback of the amplifier were all designed to achieve a low noise figure. The resulting gain and matching S-parameters are shown in Figure $7 \mathrm{~b}$, The input has a relatively high reflection coefficient as the amplifier is noise matched instead of impedance matched. This does have the effect of reflecting more than ten percent of the incoming power back out of the feed.

\subsection{Filter Amplifier}

A $60 \mathrm{~m}$ coaxial cable connects the ouput of each LNA to the sea container where each signal is received by a filter amplifier. This block comprises a custom-made Minicircuits band-pass $400-800 \mathrm{MHz}$ filter followed by 3 stages of gain. The S-parameters for this block are shown in Figure 8 . The amplifier has a flat passband with less than $3 \mathrm{~dB}$ variation. It has greater than $20 \mathrm{~dB}$ rejection by $390 \mathrm{MHz}$ on the low side and $815 \mathrm{MHz}$ on the high side. It is a highly linear device, with a measured output power compression point of $29 \mathrm{dBm}$. 


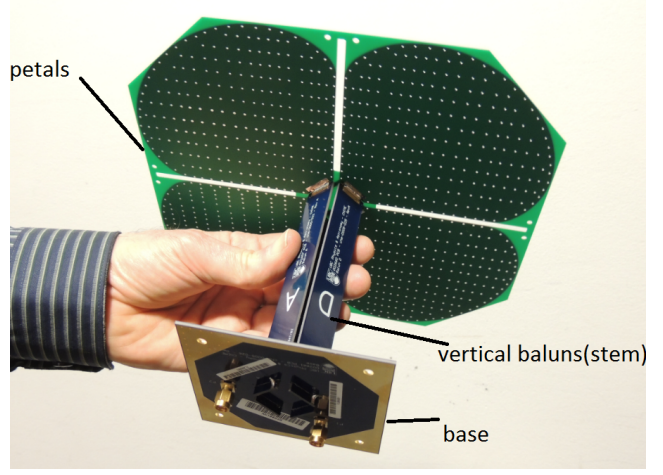

(a)

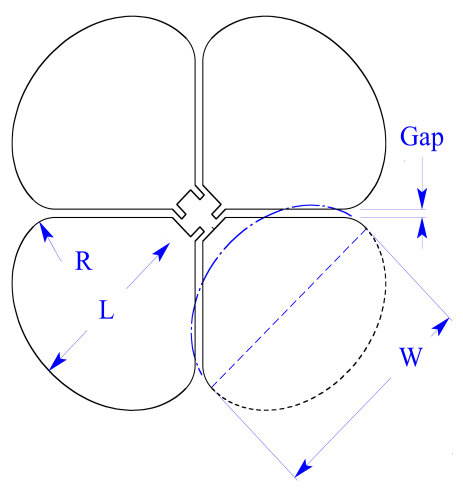

(b)

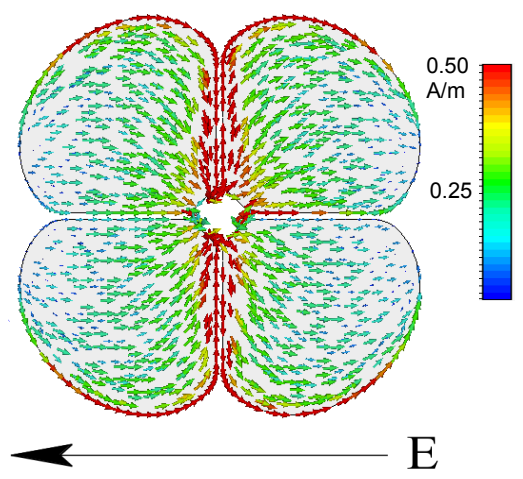

(c)

Figure 5. (a) Photo of CHIME feed; (b) Geometry of petals with $W=138.5 \mathrm{~mm}, L=131.9 \mathrm{~mm}, R=20 \mathrm{~mm}$. This geometry is small enough that a feed element is compatible with any azimuth orientation within the array. (c) The current pattern from a CST simulation of the feed at $600 \mathrm{MHz}$ for the horizontal polarization as indicated by the arrow labeled E.

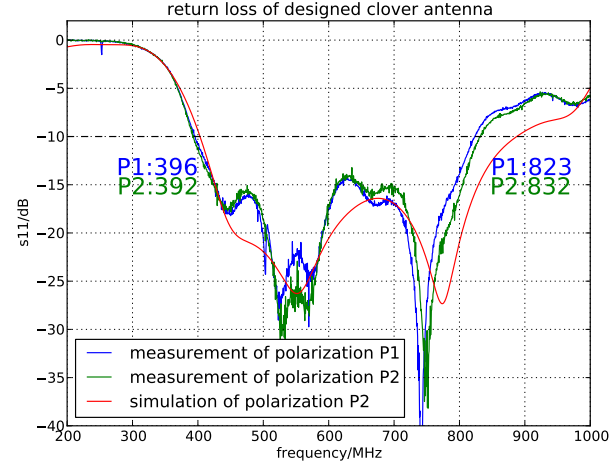

(a)

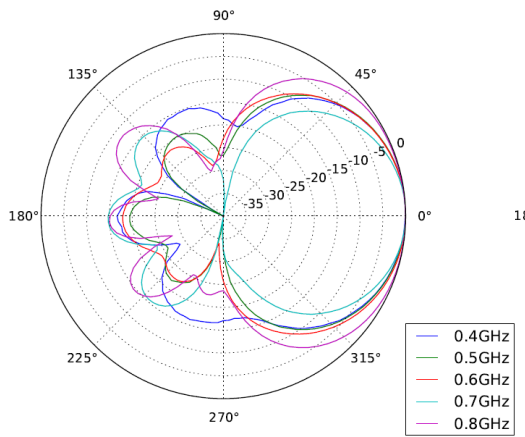

(b)

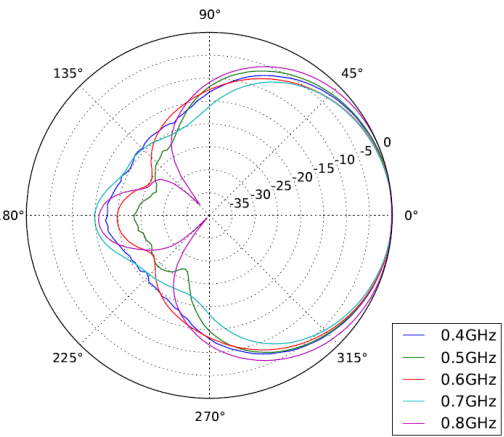

(c)

Figure 6. (a) Measured return loss compared with simulation. Note the similarity between two polarizations. (b) Measured E plane of polarization P2. (c) Measured H plane of polarization P2. 


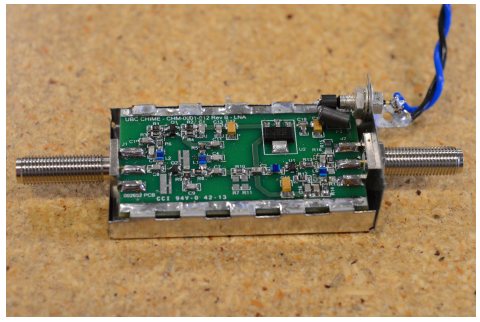

(a)

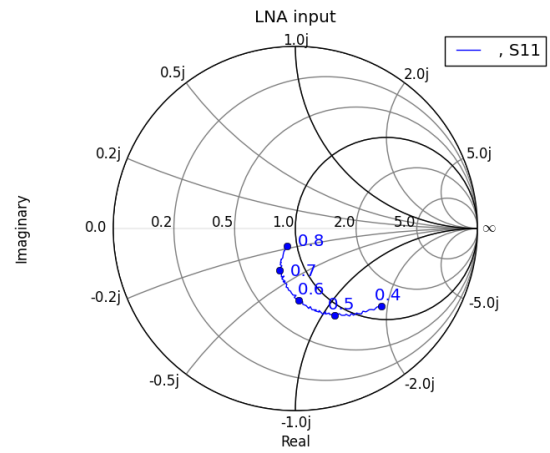

(c)

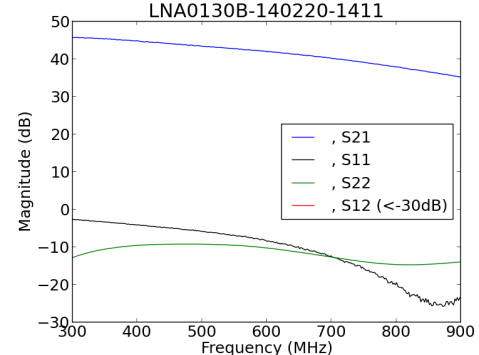

(b)

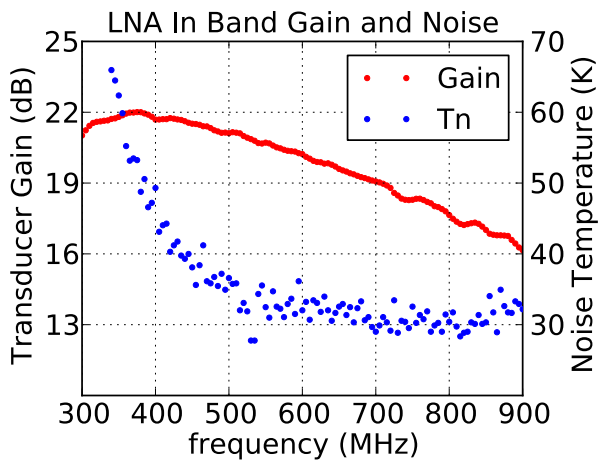

(d)

Figure 7. (a) Image of the CHIME LNA. The circuit board is soldered to the case to reduce internal resonances. (b) Measured S-parameters of the two-stage LNA. (c) Smith Chart of the LNA input matching relative to 50 ohms. (d) Measured gain and noise of the first stage of the LNA only.

\section{DIGITAL BACKEND}

The digital backend of the CHIME Pathfinder takes the overall structure of an FX correlator and is implemented into the main components shown in Figure 9.

- The analog-to-digital converters that sample the sky signal are located on daughter cards that attach to custom FPGA motherboards.

- The channelizer (F-engine) is implemented in each motherboard's FPGA to split the $400 \mathrm{MHz}$-wide analog signals into 1024 frequency bins $390 \mathrm{kHz}$ wide.

- The crossbar and shuffle modules re-organize the channelized data from all the motherboards in a crate in order to concentrate the data for a subset of frequencies into a single FPGA. A 10 Gbps full-mesh network connecting every motherboard is implemented using a passive custom backplane driven by high speed serial transceivers on the FPGA.

- The offload link packetizes the shuffled data into 10 gigabit Ethernet packets and streams those packets to the GPU correlator host computers.

- The correlator (X-engine) is implemented in a dedicated computing cluster, where each node receives the data with 10 gigabit Ethernet inputs, processes the packet header information and moves the data to system memory.

- The GPUs are used to perform efficient, real-time full $N^{2}$ correlation and averaging of the data. 


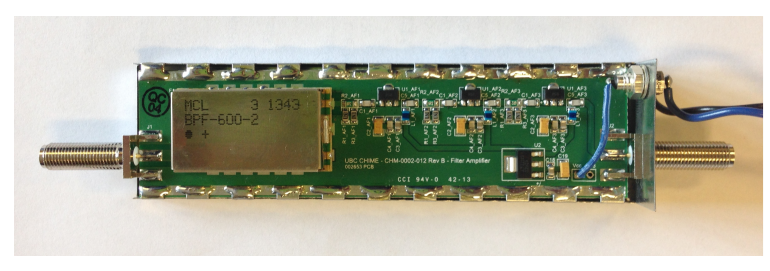

(a)

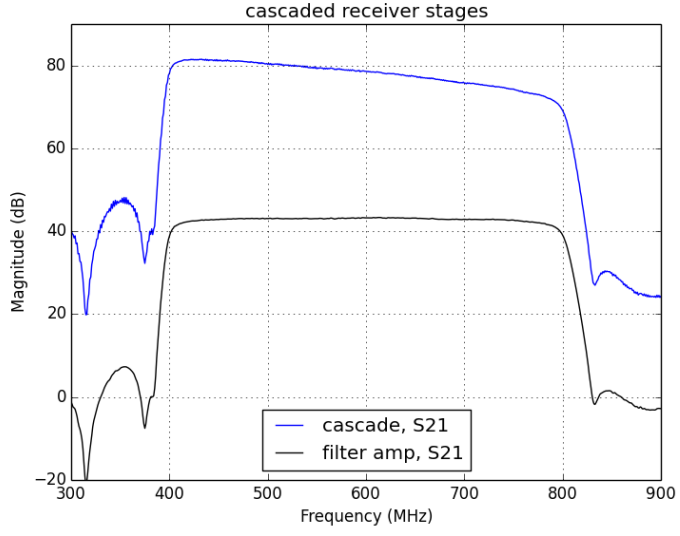

(b)

Figure 8. (a) Image of the CHIME amplifier and band-defining filter. Input on the left. (b) Gain and passband of the filter-amplifier block labeled filter amp plotted along with the full analog chain labeled cascade. The passband of the filter amplifier block is designed to be very flat with frequency. The entire analog chain has a slope from low to high frequency primarily due to the LNA gain and analog cabling.

- Commodity gigabit ethernet switches are used to collect the data onto a server which stores the integrated data. A server on this same network is used to configure and monitor the hardware in the array.

Table 2 summarizes the key design parameters of the CHIME Pathfinder's digital backend. In addition to those, the system had to be designed with enough flexibility to allow testing of real-time gain corrections, RFI removal, high-speed and triggered data tapping for ancillary science such as pulsar and radio transient signal analysis, and beamforming along each cylinder. The design is also required to be scalable to 2560 inputs for the full CHIME instrument.

The hardware, firmware and software components of the digital backend are described in more detail in the sections below.

\subsection{Analog-to-digital converter daughterboards}

The analog-to-digital conversion of the feed signals is performed using custom double-wide FPGA mezzanine card (FMC) compliant daughter boards equipped with two E2V EV8AQ160 analog-to-digital (ADC) chips (see Figure 10p. Each ADC chip has four inputs that can sample at up to 1.25 GSps at 8 bits.

The sky signal in the absence of man-made signals is well encoded with only a few bits, with 4 bits having the effect of increasing any properly amplified white noise system temperature by $\sim 2$ percent. However, man-made $\mathrm{RF}$ power consists of both broadband bursts and strong narrowband signals which require additional dynamic range. Initial testing found that 8 bit sampling will provide the dynamic range to sample the sky adequately given the RF conditions at the site.

For CHIME, the sampling rate is set to $800 \mathrm{MSps}$, and the $400-800 \mathrm{MHz}$ sky signal is directly sampled using the second Nyquist zone. The analog inputs achieve more than $15 \mathrm{~dB}$ return-loss from $300 \mathrm{MHz}$ to $1.1 \mathrm{GHz}$. The input passband is broader, from $150 \mathrm{MHz}$ to $1.1 \mathrm{GHz}$. These analog components are constrained to one section 


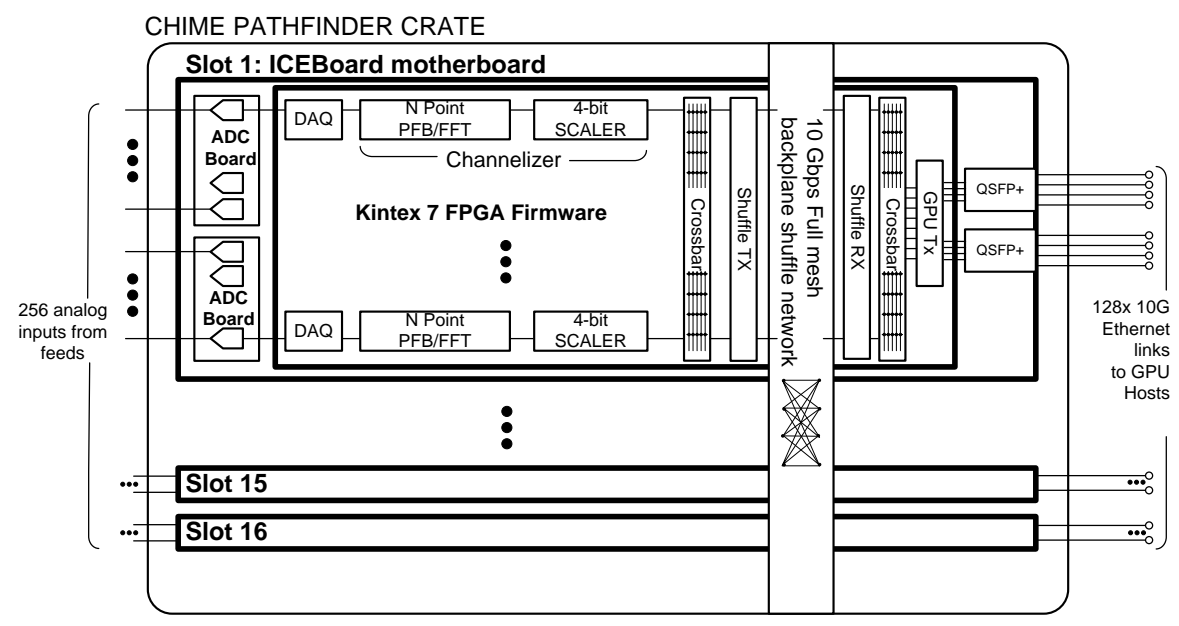

(a)

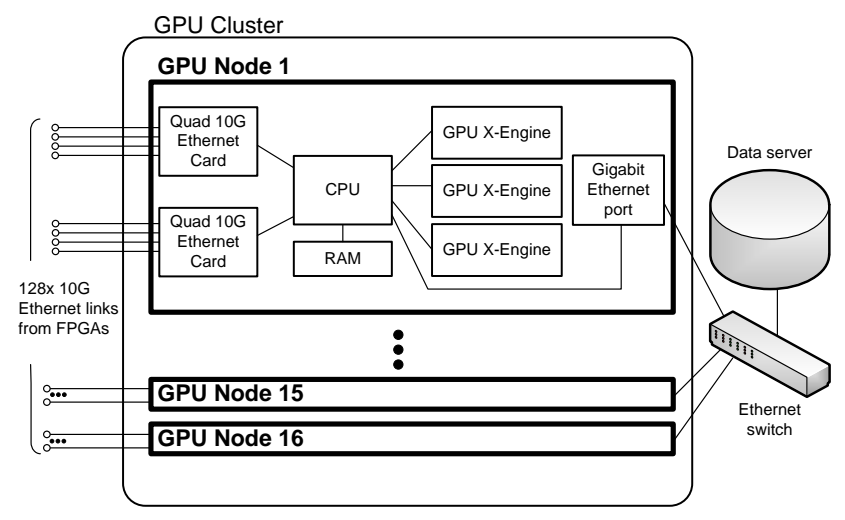

(b)

Figure 9. Digital backend system overview. a) Data is sampled by ADCs and is acquired and framed by the FPGA DAQ module, and is fed into the channelizer (F-engine) which performs a PFB/FFT and scales the data back into (4+4) bit values. The data is rearranged in the FPGA and through a high-speed backplane network and sent over 10 gigabit Ethernet to a GPU farm to be correlated. b) Diagram of Pathfinder X-engine GPU system. Data is received by two quad 10 gigabit Ethernet cards and passed into system memory. The data is then transferred in blocks to the GPU X-engine kernel for multiplication and accumulation. Finally the correlated signal is offloaded to a data server for storage and further processing.

of each board, and are behind a shield that forms a Faraday cage. The analog traces are routed predominantly on internal board layers to limit cross-talk. The neighboring inputs have been measured to have less than $-50 \mathrm{~dB}$ cross-talk levels, limited by the ADC chip.

Each daughterboard requires a $10 \mathrm{MHz}$ clock input either through a SMA connector on the front panel, or as an LVDS or LVPECL signal from the host motherboard. The board has a software-programmable phase locked loop (PLL) which creates the $1.6 \mathrm{GHz}$ clock that drives both ADCs. A post-PLL ADC clock with less than $500 \mathrm{fs}$ jitter is needed to ensure that the ADC performance is degraded by less than $0.1 \mathrm{bits}$.

A synchronization input from the motherboard or an SMA connector on the front panel allows the ADC data acquisition to be started in a precise way relative to the $10 \mathrm{MHz}$ clock and ADC clock, which allows acquisition of data frames with a deterministic phase across the array.

An on-board $\mathrm{I}^{2} \mathrm{C}$ EEPROM stores the digital serial number information along with individual board testing history, performance, and parameters. Also, an SPI bus allows the host FGPA to read out the temperatures of 


\begin{tabular}{l|l}
\hline Number of analog inputs & 256 \\
\hline Analog sampling & 800 MSps @ 8 bits \\
\hline Channelizer Type & 2048 sample PFB/FFT \\
Frequency channels & 1024 bins, $390 \mathrm{kHz} / \mathrm{bin}$ \\
Channelizer data path & Input: 8 bits \\
& Internal: $18+18$ bits complex \\
& Output: $4+4$ bits complex \\
\hline Power Consumption & Channelizer: $1.2 \mathrm{~kW}$ \\
& Correlator: $10 \mathrm{~kW}$ \\
\hline Data rates & Digitized analog inputs: 1.64 Tbps \\
& Shuffle: 1.54 Tbps (Rx+Tx, plus overhead) \\
& Output to GPU correlators: 819.2 Gbps (plus overhead) \\
& Output from GPU correlator: $\sim 100 \mathrm{Mbps}(30$ second integration) \\
\hline Baselines & 32,896 \\
\hline Computations & Channelizer: $\sim 0.6 \mathrm{~T}$ complex MAC/s \\
& Correlator: $13 \mathrm{~T}$ complex MAC/s \\
\hline
\end{tabular}

Table 2. Key parameters for the Pathfinder digital backend.

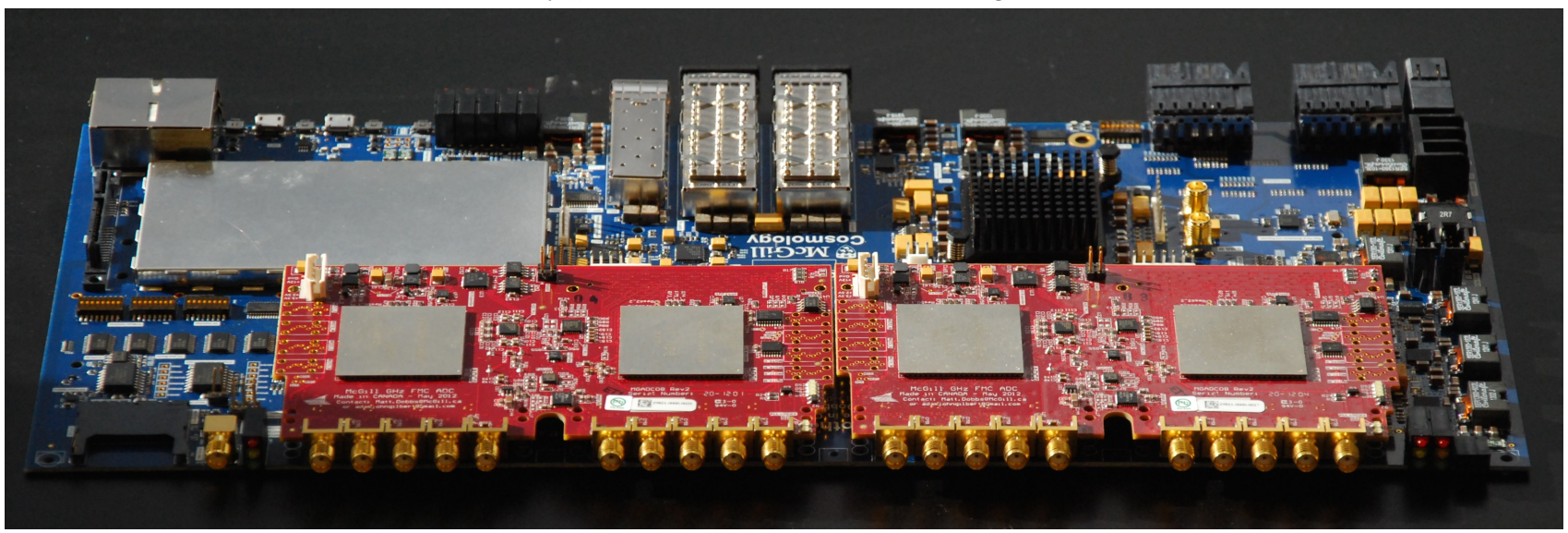

Figure 10. Two 8-channel, 8-bit, 1250 MSps, FMC-compatible analog-to-digital daughterboards (red boards) seated on a ICE motherboard (blue board).

the ADC cores and the board, as well as communicate with the ADC control registers.

\subsection{FPGA ("ICE") Motherboard}

The FPGA motherboard (also known as "ICE motherboard", shown in Figure 10) accepts two FMC-compliant high-pin-count CHIME ADC daughterboards that connect to a single Xilinx Kintex 7 XC7K420T FPGA. The choice of the FPGA was driven by requirements in terms of throughput needed for data shuffling and GPU links, Input/Output (I/O) pin count to the FMCs, backplane and other ICE motherboard subsystems, logic resources (gates, RAM, DSP blocks), cost, and power consumption.

The FPGA board has 19 high-speed (12.5 Gbps GTX) serial links interfaced through the backplane connector to support the Pathfinder and full CHIME data shuffling. It also has two QSFP+ connectors which connect to a GPU node and one SFP+ connector which connects to a control computer. The FPGA can also receive trigger, synchronization and GPS-based timestamp signals from the backplane.

The board offers an identifying EEPROM, temperature sensors, FMC power control, and voltage/current monitoring for every power rail. This allows for self discovery and diagnostics of a large set of motherboards.

Each ICE motherboard is equipped with a Texas Instruments AM3871 ARM processor with 1 gigabyte of DDR3 DRAM, an SD card slot, two gigabit Ethernet ports, USB, UART, and SATA connections. The processor 
runs a Linux operating system, allowing for remote-programming of the FPGA as well as providing always-on monitoring of the hardware and an arbitrated network-based control interface to the FPGA subsystems.

The clocking for the entire board is derived from one $10 \mathrm{MHz}$ clock which is received from the backplane. An on-board crystal oscillator and a front panel SMA connector are also available for single-board or bench top development work. The clocking is then distributed through a low-jitter network to the FMC mezzanine boards and to the two onboard PLLs which create multiple clocks to drive the FPGA and ARM processor.

The ICE motherboards are designed for a $9 \mathrm{U}$ standard VME physical crate design. They are 14 inches by 6.5 inches, and can be spaced by 0.8 inch with the ADC mezzanines installed. The FPGAs and ADCs require active cooling provided by the host crate system. Each board requires approximately $75 \mathrm{~W}$ of power when running the Pathfinder firmware with two ADC mezzanines. The board operates from a single supply in the range of $14-20 \mathrm{~V}$, and the nominal $2 \mathrm{MHz}$ buck converters can be synchronized by the FPGA to restrict the switching noise to known frequencies.

\subsection{FPGA Firmware}

The firmware that operates on the FPGA is mostly custom code written in VHDL and simulated and compiled using the latest Xilinx Vivado software suite. This approach enabled us to maximize the use of the Kintex 7 FPGA and ICE motherboard potential as the new tools could easily place, route and meet timing closure in half the time required for older Xilinx tools on large FPGA designs.

The firmware is subdivided into modules that interface with each other using the industry-standard AXI4Streaming bus protocol to carry the data and control signals.

All firmware configurations can be set talking directly to the FPGA using a simple UDP packet structure, or by communicating through the ARM processor.

The FPGA has no embedded processor and any high-level functions and algorithms are performed by custom control software on either an external control computer or the ARM processor.

In addition to the command system, the core firmware provides all the resources needed to operate the ICE motherboard independently of the ARM processor. This includes access to the buck regulators, $\mathrm{I}^{2} \mathrm{C}$-based devices, the FMC hardware, etc. An internal frequency counter monitors internal and external clocks to confirm proper operation of the signal processing chain.

The first signal processing module performs the data acquisition from the FMC ADC boards. The data is acquired at $800 \mathrm{MSps}$ through 8 LVDS lines and a $400 \mathrm{MHz}$ DDR clock. The module aligns the data acquisition of each line with a 78 ps resolution to compensate for the board and FPGA routing delays. The data is deserialized and combined into a $200 \mathrm{MHz}, 32$-bit wide AXI4 stream that is passed on to the channelizer module as frames of 2048 8-bit samples. The data acquisition module provides logic to deterministically start data framing on a known edge of the ADC sample clock relative to the $10 \mathrm{MHz}$ reference.

The channelizer module starts its signal processing by selecting its source stream from the ADC or from an integrated test pattern generator. The stream is fed to a customized poly-phase filter bank (PFB) and fastFourier transform (FFT) that has been generated by the CASPER toolset and has been wrapped in an AXI interface. The PFB includes a sinc-Hann window applied to 4 data frames, and outputs a frame of 1024 complex frequency samples in a $18+18$ bit format. The following scaler module applies a $16+16$ bit complex gain to each frequency bin. The complex gain is stored in two tables that can be configured and switched in real time in order to account for system gain and delay variations. The result is finally scaled to $(4+4)$ bit complex values and saturates instead of folding. The output data is accompanied with ADC, FFT and scaling saturation flags which can be used to identify the strongest broadband and line radio frequency interference. A FPGA-based statistics subsystem also keeps track of ADC and scaling saturations for independent data monitoring.

The data streams from the 16 channelizers are internally reordered by a crossbar module that aligns the incoming data streams and selects and routes specific frequency bins from every input to one of its 16 output streams. Each output stream is typically configured to contain a subset of 64 frequency bins from all channelizers.

\footnotetext{
${ }^{\dagger}$ https://casper.berkeley.edu/
} 
The channel selection map is fully configurable to allow exclusion of unusable frequencies (due to RFI) and to adjust the downstream bandwidth. The crossbar repacks the data and saturation flags into larger blocks to increase the efficiency of data transfers on the GPU host.

The data shuffling module takes 15 of the 16 reordered streams and sends them to every other board in the crate over the backplane using 10 Gbps links implemented using the FPGA's high-speed serial GTX transceivers. The data is encoded in $64 \mathrm{~B} / 66 \mathrm{~B}$ format, is scrambled to balance the DC content of the data, and is encapsulated in simple packets with a cyclic redundancy check (CRC) code to detect transmission errors. The data coming from the corresponding boards is also received in the same way. After the full transaction, one FPGA now possesses a subset of 64 frequency bins from all 256 channelizers of the crate.

The 16 data streams are then passed through another crossbar that rearranges the data into 8 output streams, each containing 8 frequency bins from all the channelizers of the array. An array of 8 custom 10 gigabit Ethernet UDP packet transmitters is then used to send these 8 data streams to a GPU node through the two QSFP+ connectors. Each packet is accompanied with a header identifying the data source, the format and size of the payload data, and a timestamp.

\subsection{Crate and backplane}

The CHIME Pathfinder channelizer consists of a single crate containing 16 ICE motherboards. The crate is $9 \mathrm{U}$ high and uses standard VME mechanics but a custom high-speed backplane, shown in Figure 11.

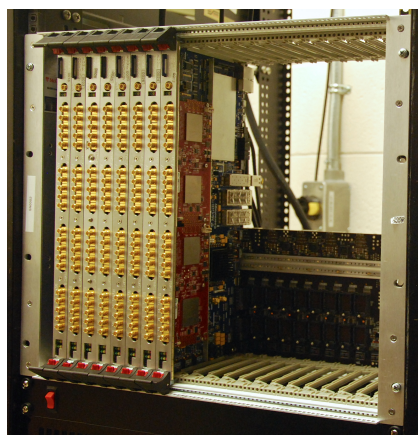

(a)

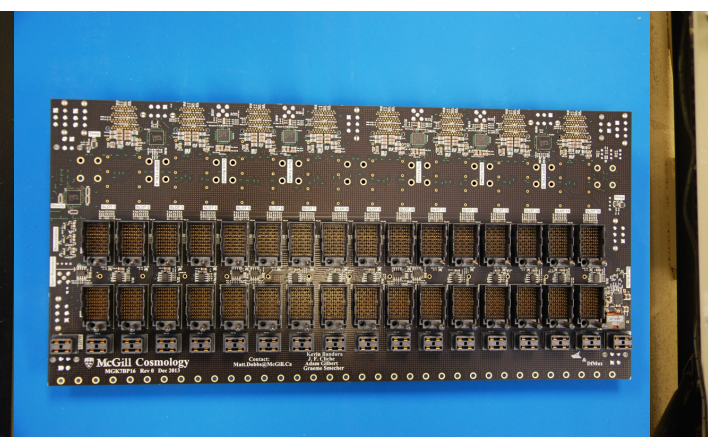

(b)

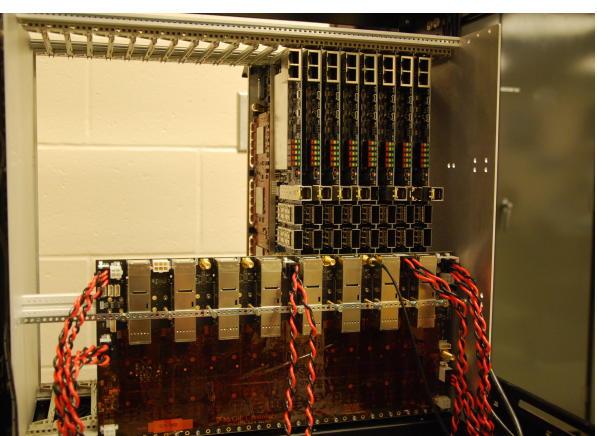

(c)

Figure 11. (a) 9U crate partially populated with 8 ICE motherboards. (b) Front image of the custom 16-slot full-mesh high-speed backplane. (c) Image of the rear of crate, showing the backplane's power entry cables, 16 QSFP+ connections and SMA inputs for clock, trigger, timestamp and synchronization distribution to the motherboards. The image also shows the rear-accessible ports provided by each ICE motherboard: 2x Gigabit Ethernet, reset buttons, USB/UART, $\mathrm{SFP}+$ and 2x QSFP+.

The backplane provides a low-jitter clock distribution system to the motherboards from a single SMA CMOS level input. It also distributes three additional digital signals: the ADC synchronization pulse, trigger pulse, and a timestamp signal. An $\mathrm{I}^{2} \mathrm{C}$ interface allows any motherboard or an external controller to remotely power down or reset any board individually, and allows the backplane temperature and power to be monitored.

A key feature of the backplane is its $10 \mathrm{Gbps}$ full-mesh networking that connects every board with every other one in both directions. This mesh connects directly to the motherboard's FPGA multi-gigabit transceivers to implement the low-cost passive shuffling network. Extreme care was taken in maximizing the signal integrity of the $10 \mathrm{Gbps}$ links. To achieve this, $25 \mathrm{Gbps}$ Molex Impact connectors (Model 761657107) are used to mate with the motherboards, and transmission line discontinuities are reduced by using single-plane direct connections and back-drilled vias. The board is made with Panasonic Megtron 6, a low-loss material compatible with more standard board assembly, and very low profile (VLP) copper foil is used to further reduce losses. The board is laid out to reduce cross-talk between signals in the network. Strong and weak signaling levels are kept well apart and are shielded by intermediate power planes. The FPGA's internal dynamic equalizer compensates for 
the low-pass frequency response and remaining distortions caused by the links. Preliminary tests between the two furthest boards (slots 1 and 16) show error-free transmission at 10 Gbps.

The backplane offers 16 QSFP+ connectors connected directly to the motherboard FPGAs to provide an additional 640 Gbps of off-crate data transfer through copper or optical cables, and will be used for the full CHIME data shuffling between 5 crates. The QSFP + can be interrogated to confirm cable connectivity and LEDs can be controlled to assist manual wiring and diagnosis.

\subsection{GPU correlator}

Visibility calculation and time averaging of all baselines takes place in a dedicated GPU-based computing cluster. A diagram of the system is shown in Figure $9 \mathrm{~b}$.

The operation is split across 16 fully independent and identical processing nodes, each responsible for processing the full set of baselines for 1/16th $(25 \mathrm{MHz})$ of the CHIME bandwidth. A single control system is housed in the same cluster, which serves the software and operating system used by the diskless nodes. This same system aggregates and buffers the data prior to long-term archiving on a data server.

Each node is housed in a $4 \mathrm{U}$ rackmount chassis and built primarily of high-end consumer-level components. The processing takes place in two AMD r9 280x GPUs and one r9 270x GPU. A pair of enterprise network interface cards (NICs) receives a total of eight 10 gigabit network connections, streaming a total of 51.2 Gbps of radiometric data, along with associated headers and flags. This data rate sets the requirements for most system components.

The incoming data is transferred over a third generation PCIe bus ( 8 lanes for each of the network boards) into primary system memory. Headers and flags are stripped from the data for further processing. Each packet header has a sequence number which allows the system to track and manage packet loss, and a stream ID identifies the frequencies in the packet. The sequence number is identical across all links for a given ADC sampling period, so it is used to provide timing and synchronization between hosts. The flags are used to track and correct for ADC and scalar overflows in the data samples.

The data is then DMA transferred from system memory into GPU buffers in large (256MB) blocks. Each GPU hosts $3 \mathrm{~GB}$ of on-board RAM (2 GB for the 270x), used to buffer incoming data prior to processing. For maximum computational flexibility, 3 connections are distributed to each r9 280x, 2 connections to the r9 270xthis results in all boards operating at roughly $2 / 3$ utilization while performing the $N^{2}$ correlation. The transfer is controlled by an Intel i7-4820k CPU, allowing 40 total lanes of PCIe-3 communications. An EVGA x79 Dark motherboard was chosen to allow 8 lanes to each of the 5 expansion boards (2xNIC, 2x r9 280x, 1x r9 270x). A primary bottleneck in the system was found to be the CPU-memory interconnect, and DDR3 $2133 \mathrm{MHz}$ overclocked RAM is used to maximize performance.

The correlation operation takes place in a custom processing kernel written in the OpenCL languag $\AA^{\mp}$ Details of this kernel will be presented in a future paper; we describe it briefly here. A single instance of the kernel (an OpenCL "Work Item," WI) computes 4x4 correlations and accumulates them over 256 time steps, roughly $0.6 \mathrm{~ms}$. These WIs are grouped into sets of 64 (OpenCL "Work Groups") which share high-speed local memory and compute a $32 \times 32$ correlation block. The 256x256 matrix of correlations is divided into these $32 \times 32$ sub-blocks, and the 36 upper-triangle blocks are computed and accumulated. (The remaining 28 blocks contain no additional information, due to the symmetry of correlations.) Efficient operation requires that all calculations be pipelined as multiply-accumulate operations (MACs), and the algorithm is able to operate efficiently by using integer operations and packing two 4-bit values into each register. This packing sets the 256-timestep accumulation period, and requires a handful of book-keeping operations to take place at the end of each MAC loop, accounting for the 8 least significant bit (LSB) offset on each sample, and accumulating the real and complex portions into 32-bit buffers. Multiple kernel invocations result in longer accumulations, with correlation buffers read out for archiving at 10-30s cadence.

The control system collects the output correlations over a gigabit Ethernet network, merges the data and archives it locally to an array of 3TB hard drives. This array can buffer at most several days of data, with the

\footnotetext{
${ }^{\ddagger}$ https://www.khronos.org/opencl/
} 
long-term archive stored in another local building, on a much larger array of disks. Custom-written software registers basic information about data products in a MySQL database for indexing purposes. The same software also manages automatic transfer of data products between acquisition computers and long-term storage and analysis nodes.

Cooling of the GPUs is a significant concern. Consumer-level GPUs are assembled and sold by a variety of vendors (using the same basic layout and identical processors), with various solutions for heat dissipation. A variety of brands were tested for thermal performance, and Sapphire "Dual-X" branded boards were chosen for both the r9 280x and r9 270x, due to their remarkable cooling capabilities (keeping the processor die to roughly $70 \mathrm{C}$ under load, $20-30 \mathrm{C}$ cooler than all other models tested in our setup). We are presently exploring direct-to-chip watercooling options, and anticipate retrofitting the system in the coming months.

\section{OBSERVATION STRATEGIES AND CHALLENGES}

The CHIME Pathfinder observing strategy is quite simple, since the instrument cannot move. Each day the telescope observes the whole northern sky, with the observation time for every source set by the east-west field of view of $2.5^{\circ}-1.3^{\circ}$ and the source declination: obstime/day $=4 \mathrm{~min} \times$ fov $\times \cos (\mathrm{dec})$. The full correlation matrix is saved at a 30 second cadence.

One of the challenges of the system comes from the calibration needed in order to detect the BAO with the CHIME Pathfinder. From previous simulations of a Pathfinder-like instrument $\frac{15}{15}$ all telescope primary beams must be known to $0.1 \%$ and the system gain for each feed must be known to $1 \%$ in order to be able to properly reconstruct the power spectrum. See the accompanying SPIE proceedings ${ }^{14}$ for full details about the calibration of CHIME, which will be implemented on the Pathfinder. A brief summary of the calibration procedures planned to achieve these levels of instrument stability is given below.

- Measure the primary beam using bright point sources and pulsars. The bright points sources will give a first measure of an overall complex gain calibration and beam-width as a function of frequency. Pulsars with their inherent on-off period allow one to remove all signals from that data that do not pulse at the frequency of the pulsar. The beam will be measured further using pulsar holography by additionally correlating the signal from the DRAO $26 \mathrm{~m}$ Telescope tracking the pulsars.

- Inject a broadband calibration signal. We will inject into every feed a broadband calibration signal, and measure and correlate that signal with all the CHIME feeds. This low-level injected signal is switched on and off on a $\sim$ second timescale. The switched signal is then used to measure and adjust for the complex relative gain of every feed, removing complex gain changes on scales longer than the switching time.

- Use the redundant baseline information. The CHIME Pathfinder is a very redundant interferometer by design. These redundant baselines can be used to calculate the complex gain of each feed without having any previous knowledge of the sky.

Another remaining challenge lies in interference management. Even though the CHIME Pathfinder is located in the radio astronomy reserve of the Dominion Radio Astrophysical Observatory, there are still man-made radio frequency signals which will interfere with observing the sky. The CHIME Pathfinder plans to use real-time RFI flagging and excision techniques to mitigate those. The most extreme events which saturate the digital signal are flagged by the FPGA system and are passed to the GPU X-engine. Further processing will be performed on an intermediate integration scale of milliseconds to further process the data looking for excessive excursions of the signal. Post-processing will handle the final data clean-up.

\section{CONCLUSION}

The CHIME Pathfinder is currently being commissioned at the Dominion Radio Astrophysical Observatory. The cylinder structures have been completed and the analog and digital electronics are being phased in with 16 feed channels currently installed. The first fringes observed with the telescope, from Cassiopia A, are shown in Figure 12. We are comparing measured sky maps to our galactic model to assess system temperature. We have 


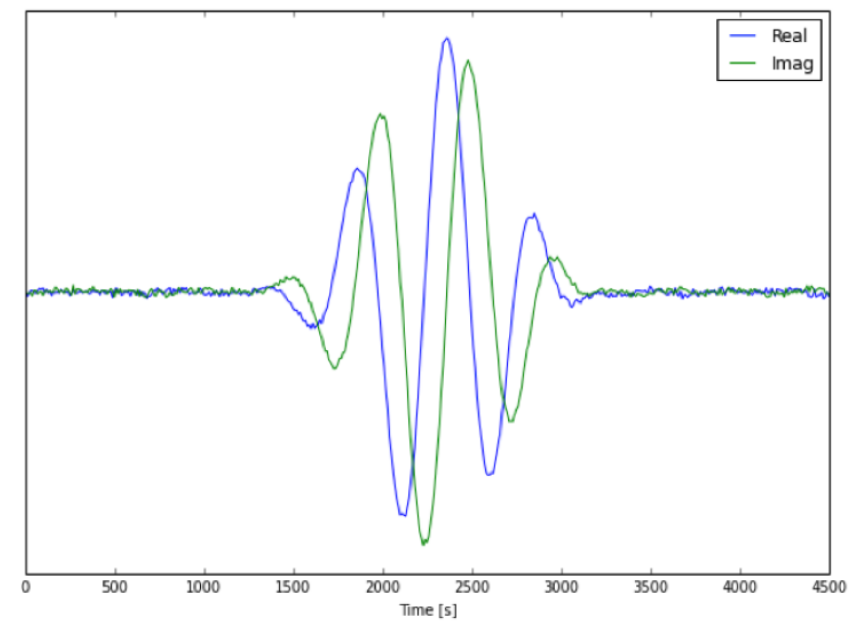

Figure 12. First fringes from an east-west CHIME Pathfinder baseline of . Shown are the real and imaginary parts of a single visibility for a single frequency channel.

performed holography with the DRAO $26 \mathrm{~m}$ telescope to measure our beam shape. Finally, we have installed a broadband signal injection system to monitor complex gain in real-time.

The CHIME Pathfinder is paving the way for all the aspects of the CHIME experiment. It is a $1 / 7$ th area version of the full system with $1 / 10$ th of the analog and digital electronics. All components being tested are to be scaled up to the full system. The Pathfinder will also be used to explore beyond the baseline design by investigating RF-over-fiber links, $\frac{23}{23}$ and real-time FFT beam-forming with the FPGAs and GPUs. Meanwhile, ground is currently being broken in preparation for the full CHIME construction.

\section{ACKNOWLEDGMENTS}

We are very grateful for the warm reception and skillful help we have received from the staff of the Dominion Radio Astrophysical Observatory, operated by the National Research Council Canada.

We acknowledge support from the Canada Foundation for Innovation, the Natural Sciences and Engineering Research Council of Canada, the B.C. Knowledge Development Fund, le Cofinancement gouvernement du Québec-FCI, the Ontario Research Fund, the CIfAR Cosmology and Gravity program, the Canada Research Chairs program, and the National Research Council of Canada. M. Deng acknowledges a MITACS fellowship. We thank Xilinx and the XUP for their generous donations.

\section{REFERENCES}

1. Weinberg, D. H., Mortonson, M. J., Eisenstein, D. J., Hirata, C., Riess, A. G., and Rozo, E., "Observational probes of cosmic acceleration," Physics Reports 530, 87-255 (Sept. 2013).

2. Loeb, A. and Wyithe, J. S. B., "Possibility of Precise Measurement of the Cosmological Power Spectrum with a Dedicated Survey of 21cm Emission after Reionization," Physical Review Letters 100, 161301 (Apr. 2008).

3. Eisenstein, D. J., Zehavi, I., Hogg, D. W., Scoccimarro, R., Blanton, M. R., Nichol, R. C., Scranton, R., Seo, H.-J., Tegmark, M., Zheng, Z., Anderson, S. F., Annis, J., Bahcall, N., Brinkmann, J., Burles, S., Castander, F. J., Connolly, A., Csabai, I., Doi, M., Fukugita, M., Frieman, J. A., Glazebrook, K., Gunn, J. E., Hendry, J. S., Hennessy, G. S., Ivezić, Ž., Kent, S., Knapp, G. R., Lin, H., Loh, Y.-S., Lupton, R. H., Margon, B., McKay, T. A., Meiksin, A., Munn, J. A., Pope, A., Richmond, M. W., Schlegel, D. J., Schneider, D. P., Shimasaku, K., Stoughton, C., Strauss, M. A., SubbaRao, M., Szalay, A. S., Szapudi, I., Tucker, D. L., Yanny, B., and York, D. G., "Detection of the Baryon Acoustic Peak in the Large-Scale Correlation Function of SDSS Luminous Red Galaxies," The Astrophysical Journal 633, 560-574 (Nov. 2005). 
4. Cole, S., Percival, W. J., Peacock, J. A., Norberg, P., Baugh, C. M., Frenk, C. S., Baldry, I., Bland-Hawthorn, J., Bridges, T., Cannon, R., Colless, M., Collins, C., Couch, W., Cross, N. J. G., Dalton, G., Eke, V. R., de Propris, R., Driver, S. P., Efstathiou, G., Ellis, R. S., Glazebrook, K., Jackson, C., Jenkins, A., Lahav, O., Lewis, I., Lumsden, S., Maddox, S., Madgwick, D., Peterson, B. A., Sutherland, W., and Taylor, K., "The 2dF Galaxy Redshift Survey: power-spectrum analysis of the final data set and cosmological implications," Monthly Notices of the Royal Astronomical Society 362, 505-534 (Sept. 2005).

5. Blake, C., Kazin, E. A., Beutler, F., Davis, T. M., Parkinson, D., Brough, S., Colless, M., Contreras, C., Couch, W., Croom, S., Croton, D., Drinkwater, M. J., Forster, K., Gilbank, D., Gladders, M., Glazebrook, K., Jelliffe, B., Jurek, R. J., Li, I.-H., Madore, B., Martin, D. C., Pimbblet, K., Poole, G. B., Pracy, M., Sharp, R., Wisnioski, E., Woods, D., Wyder, T. K., and Yee, H. K. C., "The WiggleZ Dark Energy Survey: mapping the distance-redshift relation with baryon acoustic oscillations," Monthly Notices of the Royal Astronomical Society 418, 1707 (2011).

6. Anderson, L., Aubourg, É., Bailey, S., Beutler, F., Bhardwaj, V., Blanton, M., Bolton, A. S., Brinkmann, J., Brownstein, J. R., Burden, A., Chuang, C.-H., Cuesta, A. J., Dawson, K. S., Eisenstein, D. J., Escoffier, S., Gunn, J. E., Guo, H., Ho, S., Honscheid, K., Howlett, C., Kirkby, D., Lupton, R. H., Manera, M., Maraston, C., McBride, C. K., Mena, O., Montesano, F., Nichol, R. C., Nuza, S. E., Olmstead, M. D., Padmanabhan, N., Palanque-Delabrouille, N., Parejko, J., Percival, W. J., Petitjean, P., Prada, F., PriceWhelan, A. M., Reid, B., Roe, N. A., Ross, A. J., Ross, N. P., Sabiu, C. G., Saito, S., Samushia, L., Sánchez, A. G., Schlegel, D. J., Schneider, D. P., Scoccola, C. G., Seo, H.-J., Skibba, R. A., Strauss, M. A., Swanson, M. E. C., Thomas, D., Tinker, J. L., Tojeiro, R., Magana, M. V., Verde, L., Wake, D. A., Weaver, B. A., Weinberg, D. H., White, M., Xu, X., Yeche, C., Zehavi, I., and Zhao, G.-B., "The clustering of galaxies in the SDSS-III Baryon Oscillation Spectroscopic Survey: baryon acoustic oscillations in the Data Releases 10 and 11 Galaxy samples," Monthly Notices of the Royal Astronomical Society 441, 24-62 (June 2014).

7. Slosar, A., Iršič, V., Kirkby, D., Bailey, S., Busca, N. G., Delubac, T., Rich, J., Aubourg, É., Bautista, J. E., Bhardwaj, V., Blomqvist, M., Bolton, A. S., Bovy, J., Brownstein, J., Carithers, B., Croft, R. A. C., Dawson, K. S., Font-Ribera, A., Le Goff, J. M., Ho, S., Honscheid, K., Lee, K.-G., Margala, D., McDonald, P., Medolin, B., Miralda-Escude, J., Myers, A. D., Nichol, R. C., Noterdaeme, P., PalanqueDelabrouille, N., Pâris, I., Petitjean, P., Pieri, M. M., Piškur, Y., Roe, N. A., Ross, N. P., Rossi, G., Schlegel, D. J., Schneider, D. P., Suzuki, N., Sheldon, E. S., Seljak, U., Viel, M., Weinberg, D. H., and Yeche, C., "Measurement of baryon acoustic oscillations in the Lyman- $\alpha$ forest fluctuations in BOSS data release 9," Journal of Cosmology and Astroparticle Physics 04, 026 (Apr. 2013).

8. Busca, N. G., Delubac, T., Rich, J., Bailey, S., Font-Ribera, A., Kirkby, D., Le Goff, J. M., Pieri, M. M., Slosar, A., Aubourg, E., Bautista, J. E., Bizyaev, D., Blomqvist, M., Bolton, A. S., Bovy, J., Brewington, H., Borde, A., Brinkmann, J., Carithers, B., Croft, R. A. C., Dawson, K. S., Ebelke, G., Eisenstein, D. J., Hamilton, J. C., Ho, S., Hogg, D. W., Honscheid, K., Lee, K. G., Lundgren, B., Malanushenko, E., Malanushenko, V., Margala, D., Maraston, C., Mehta, K., Miralda-Escude, J., Myers, A. D., Nichol, R. C., Noterdaeme, P., Olmstead, M. D., Oravetz, D., Palanque-Delabrouille, N., Pan, K., Pâris, I., Percival, W. J., Petitjean, P., Roe, N. A., Rollinde, E., Ross, N. P., Rossi, G., Schlegel, D. J., Schneider, D. P., Shelden, A., Sheldon, E. S., Simmons, A., Snedden, S., Tinker, J. L., Viel, M., Weaver, B. A., Weinberg, D. H., White, M., Yèche, C., and York, D. G., "Baryon acoustic oscillations in the Ly $\alpha$ forest of BOSS quasars," Astronomy and Astrophysics 552, 96 (Apr. 2013).

9. Chang, T.-C., Pen, U.-L., Bandura, K., and Peterson, J. B., "An intensity map of hydrogen 21-cm emission at redshift z 0.8," Nature 466, 463-465 (July 2010).

10. Switzer, E. R., Masui, K. W., Bandura, K., Calin, L. M., Chang, T. C., Chen, X. L., Li, Y. C., Liao, Y. W., Natarajan, A., Pen, U.-L., Peterson, J. B., Shaw, J. R., and Voytek, T. C., "Determination of z 0.8 neutral hydrogen fluctuations using the $21 \mathrm{~cm}$ intensity mapping autocorrelation," Monthly Notices of the Royal Astronomical Society: Letters, L125 (June 2013).

11. Masui, K. W., Switzer, E. R., Banavar, N., Bandura, K., Blake, C., Calin, L. M., Chang, T. C., Chen, X., Li, Y. C., Liao, Y. W., Natarajan, A., Pen, U.-L., Peterson, J. B., Shaw, J. R., and Voytek, T. C., "Measurement of $21 \mathrm{~cm}$ Brightness Fluctuations at z 0.8 in Cross-correlation," The Astrophysical Journal Letters 763, L20 (Jan. 2013). 
12. Hinshaw, G., Larson, D., Komatsu, E., Spergel, D. N., Bennett, C. L., Dunkley, J., Nolta, M. R., Halpern, M., Hill, R. S., Odegard, N., Page, L., Smith, K. M., Weiland, J. L., Gold, B., Jarosik, N., Kogut, A., Limon, M., Meyer, S. S., Tucker, G. S., Wollack, E., and Wright, E. L., "Nine-year Wilkinson Microwave Anisotropy Probe (WMAP) Observations: Cosmological Parameter Results," Astrophysical Journal Supplement Series 208, 19 (Oct. 2013).

13. Planck Collaboration, Ade, P. A. R., Aghanim, N., Armitage-Caplan, C., Arnaud, M., Ashdown, M., Atrio-Barandela, F., Aumont, J., Baccigalupi, C., Banday, A. J., and et al., "Planck 2013 results. XVI. Cosmological parameters," ArXiv e-prints (Mar. 2013).

14. Newburgh, L. et al., "Calibrating CHIME: A New Radio Interferometer to Probe Dark Energy," in [Society of Photo-Optical Instrumentation Engineers (SPIE) Conference Series], Society of Photo-Optical Instrumentation Engineers (SPIE) Conference Series (2014). In preparation.

15. Shaw, J. R., Sigurdson, K., Sitwell, M., Stebbins, A., and Pen, U.-L., "Coaxing Cosmic 21cm Fluctuations from the Polarized Sky using m-mode Analysis," arXiv.org (Jan. 2014).

16. Shaw, J. R., Sigurdson, K., Pen, U.-L., Stebbins, A., and Sitwell, M., "All-sky Interferometry with Spherical Harmonic Transit Telescopes," The Astrophysical Journal 781, 57 (Feb. 2014).

17. Lorimer, D. R., Bailes, M., McLaughlin, M. A., Narkevic, D. J., and Crawford, F., "A Bright Millisecond Radio Burst of Extragalactic Origin," Science 318, 777 (Nov. 2007).

18. Thornton, D., Stappers, B., Bailes, M., Barsdell, B., Bates, S., Bhat, N. D. R., Burgay, M., Burke-Spolaor, S., Champion, D. J., Coster, P., D’Amico, N., Jameson, A., Johnston, S., Keith, M., Kramer, M., Levin, L., Milia, S., Ng, C., Possenti, A., and van Straten, W., "A Population of Fast Radio Bursts at Cosmological Distances," Science 341, 53-56 (July 2013).

19. Spitler, L. G., Cordes, J. M., Hessels, J. W. T., Lorimer, D. R., McLaughlin, M. A., Chatterjee, S., Crawford, F., Deneva, J. S., Kaspi, V. M., Wharton, R. S., Allen, B., Bogdanov, S., Brazier, A., Camilo, F., Freire, P. C. C., Jenet, F. A., Karako-Argaman, C., Knispel, B., Lazarus, P., Lee, K. J., van Leeuwen, J., Lynch, R., Lyne, A. G., Ransom, S. M., Scholz, P., Siemens, X., Stairs, I. H., Stovall, K., Swiggum, J. K., Venkataraman, A., Zhu, W. W., Aulbert, C., and Fehrmann, H., "Fast Radio Burst Discovered in the Arecibo Pulsar ALFA Survey," arXiv.org, 2934 (Apr. 2014).

20. Feng, L., Vaulin, R., and Hewitt, J. N., "Detectability of Late-Time Radio Afterglows from Compact Binary Coalescence," arXiv.org (May 2014).

21. Leung, M., A Wideband Feed for a Cylindrical Radio Telescope, PhD thesis, University of Sydney (May 2008).

22. Deng, M. and Campbell-Wilson, D., "The Cloverleaf Antenna: A Compact Wide-bandwidth Dualpolarization Feed for CHIME," ANTEM 2014 (2014). In preparation.

23. Mena, J., Bandura, K., Cliche, J. F., Dobbs, M., Gilbert, A., and Tang, Q. Y., "A Radio-Frequencyover-Fiber link for large-array radio astronomy applications," Journal of Instrumentation 8, 10003 (Oct. 2013). 